\title{
Circular Dichroism of Gold Bipyramid Dimers
}

Radosław Deska, Patryk Obstarczyk, Katarzyna Matczyszyn, Joanna Olesiak-Bańska*

Advanced Materials Modelling and Engineering Group, Wroclaw University of Science and

Technology, Poland

\section{SUPPORTING INFORMATION}

\section{BP geometry definition}

A single particle geometry was built as two cones joint at the base and rounded at the tips and in the joining region ("bulk" in the table below). The initial dimensions are defined through final particle dimensions (which are known from TEM imaging) and rounded as described in the table below (in parentheses the values for the "smaller" bipyramids were given):

\begin{tabular}{l|c|l}
$\begin{array}{l}\text { parameter } \\
\text { name }\end{array}$ & expression & description \\
\hline 10 & $102(68)[\mathrm{nm}]$ & nanoparticle final length \\
\hline $\mathrm{rtx}$ & $15(10)[\mathrm{nm}]$ & length loss after tip round \\
\hline $\mathrm{L}$ & $10+2 \cdot \mathrm{rtx}$ & NP initial length \\
\hline $\mathrm{d} 0$ & $36(24)[\mathrm{nm}]$ & NP final diameter \\
\hline $\mathrm{rbx}$ & $0.28(0.1866)[\mathrm{nm}]$ & diameter loss after tip \\
\hline $\mathrm{D}$ & $\frac{\mathrm{d} 0+2 \cdot \mathrm{rbx}}{\mathrm{rtx}}$ & NP initial diameter \\
\hline $\mathrm{rtip}$ & $\sqrt{\frac{\mathrm{D}^{2}+\mathrm{L}^{2}}{\mathrm{~L}}-1}$ & tip roundness radius \\
\hline rbulk & $\frac{\mathrm{rbx}}{\sqrt{\frac{\mathrm{D}^{2}+\mathrm{L}^{2}}{\mathrm{~L}}-1}}$ & bulk roundness radius
\end{tabular}




\section{Description of modelled physics}

Using COMSOL Multiphysics 5.0.1.276, we solve Electromagnetic (EM) and Heat Transfer (HT) problem in Frequency-Stationary mode, that is the Maxwell equations are solved in the frequency domain and the HT equation is in the stationary form. We also assume no mass exchange in the model.

$$
\begin{gathered}
\nabla \times \mu_{r}^{-1}(\nabla \times \boldsymbol{E})-k_{0}^{2}\left(\epsilon_{r}-\frac{j \sigma}{\omega \epsilon_{0}}\right) \boldsymbol{E}=0 \\
\nabla \cdot(-k \nabla T)=\frac{1}{2} \operatorname{Re}\left(\boldsymbol{J} \cdot \boldsymbol{E}^{\star}\right)+\frac{1}{2} \operatorname{Re}\left(i \omega \boldsymbol{B} \cdot \boldsymbol{H}^{\star}\right)
\end{gathered}
$$

Total electric field, E, is the sum of incident (background) and scattered (relative) field. A general incident field is defined as a plane wave travelling in the $+z$ direction:

$$
\begin{gathered}
E_{b, x}=E_{b} \cdot \cos \left(\psi_{0}\right) \exp (-\mathrm{j} \cdot k z) \exp (\mathrm{j} \cdot 2 \chi) \\
E_{b, y}=E_{b} \cdot \sin \left(\psi_{0}\right) \exp (-\mathrm{j} \cdot k z)
\end{gathered}
$$

- linear polarization $\left(\chi=0\right.$ and $\left.\psi_{0}=[0, \pi)\right)$

- circular polarization

- left-handed, or counter-rotating as defined from the point of view of the source:

$$
\psi_{0}=\frac{\pi}{4}, \chi=-\frac{\pi}{4}
$$

○ right-handed:

$$
\psi_{0}=\frac{\pi}{4}, \chi=+\frac{\pi}{4}
$$

The models were limited by Perfectly Matched Layer as a domain totally absorbing scattered field. The scattered wave was assumed to be a spherical wave sourcing from $(0,0,0)$. Wave equations were solved separately for the nanoparticle(s), and the surrounding medium (also the substrate, if applicable).

In our model gradient of refractive index in surrounding water is considered. To solve in water domains, $\epsilon_{r}=n(\lambda, T)^{2}$ was taken as a function based on Bashkatov's and Genina's formula ${ }^{1}$ containing information on both thermal and spectral dependence of water refractive index. The fitting parameters in this formula have been optimized to better reflect the data. ${ }^{2-3}$

$$
\begin{gathered}
n(\lambda, T)=A(T)+\frac{B(T)}{\lambda^{2}}+\frac{C(T)}{\lambda^{4}}+\frac{D(T)}{\lambda^{6}} \\
A(T)=1.32151-1.02801 \cdot 10^{-5} T-1.83882 \cdot 10^{-6} T^{2}+4.87625 \cdot 10^{-9} T^{3} \\
B(T)=5244.54296-2.39947 T-0.044 T^{2}+1.46191 \cdot 10^{-4} T^{3} \\
C(T)=-3.14031 \cdot 10^{8}+290367.7585 T+8.5412 \cdot 10^{2} T^{2}+1.10952 T^{3} \\
D(T)=5.18511 \cdot 10^{12}-3.52388 \cdot 10^{9} T-3.57241 \cdot 10^{7} T^{2}-4.31619 \cdot 10^{4} T^{3}
\end{gathered}
$$

where $\lambda$ is given in $[\mathrm{nm}]$ and $\mathrm{T}$ is given in $[\operatorname{deg} \mathrm{C}]$.

To solve in gold domains, complex relative permittivity values were taken from Johnson and Christy ${ }^{4}$. Heat capacity at constant pressure was taken from Sassaroli et al. ${ }^{5}$ We simulate nanoparticles deposited on glass, with relative permittivity, thermal conductivity and heat capacity at constant pressure taken from Lide's CRC Handbook². 
Electromagnetic wave absorption, or dissipation, comes from resistive heating and magnetic losses within a material, therefore absorption cross-section can be defined as ratio between integral of power loss density over the absorbing volume, $Q_{h}$, [W] and incident energy flux, $I_{i n c},\left[\mathrm{~W} / \mathrm{m}^{2}\right]$ (explicitly input as 1 $\left.\left[\mathrm{GW} / \mathrm{m}^{2}\right]\right)^{6}$ :

$$
\sigma_{a b s}=\frac{\int_{V} Q_{h}}{I_{\text {inc }}}
$$

We calculate absorption cross-section in the nanoparticles' domains only, since the electromagnetic loss due to conductivity of gold is of many orders of magnitude larger than the losses in the surrounding media.

Scattering cross-section is defined as ratio between scattered power $[\mathrm{W}]$ to incident energy flux $\left[\mathrm{W} / \mathrm{m}^{2}\right]$ (calculated as the integral of squared ratio between scattering amplitude norm, $\boldsymbol{E}_{\boldsymbol{f} \boldsymbol{f}}$, and background amplitude, $E_{b}$, referred to far-field transform sphere surface of radius $\left.r_{f f}\right)$ :

$$
\sigma_{s c a}=r_{f f}^{-2} \cdot \boldsymbol{r}_{\mathbf{0}}^{2} \cdot \int_{S}\left(\frac{\boldsymbol{E}_{\boldsymbol{f f}}}{E_{b}}\right)^{2} d S
$$

\section{Methods}

We used commercial finite-element method (FEM) software, COMSOL Multiphysics 5.0.1.276, to perform simulations of gold BPs based on TEM images of synthetically prepared bipyramids (Figure S2 and Figure S3 in supplementary information). The bipyramids were modelled as bicones with tips and central part rounded. We calculated extinction spectra of the BP dimers for left-handed and righthanded circularly polarized light (LCP and RCP, respectively) with a distance, $d$, and the yaw angle, $\theta$, between the constituent nanoparticles, see Figure 1 in the main text. The distance is defined in the projection plane perpendicular to incident beam as a gap between curved tip of one BP and the other $\mathrm{BP}$ side. The distance and the yaw angle vary in the range $5-25 \mathrm{~nm}$ and $31-180^{\circ}$, respectively, where the minimal angle $31^{\circ}$ results from $\mathrm{BP}$ aspect ratio (AR), based on initial (non-rounded) geometry of BP (see Section 1 above). The angular dependence was solved for the distance of $5 \mathrm{~nm}$. The distance dependence was solved for the angle of $45^{\circ}$. We consider the bipyramids lying on a glass surface with the long axis of each BP pitched at $31^{\circ}$ to the surface (again, this angle results from the aspect ratio; the angle was not changed during our simulations of chirality), yielding adjacency of BP's one side to the surface to reflect most probable positionings of single bipyramid on surface (cf. Up-Up, Up-Down, Down-Down configurations by Sivun et al. ${ }^{7}$ ). From the computation solutions we evaluated: absorption cross-section (resistive and magnetic power loss divided by incident irradiance that was set at $1 \mathrm{GW} / \mathrm{m}^{2}$ ), scattering cross-section (far-field radiation power normalized by the surface of far-field transform sphere and divided by incident irradiance), and extinction cross-section (the sum of absorption and scattering cross-sections). We then calculated a dissymmetry g-factor for each cross-section, which defines the unitless circular dichroism as the LCP $\left(\sigma_{L C P}\right)$ and RCP $\left(\sigma_{R C P}\right)$ cross-section difference normalized by 
average cross-section of LCP and RCP, which allows for comparison of different optical systems. For details of modelled physics, see Section 2 above.

$$
g(\lambda)=\frac{2\left(\sigma_{L C P}(\lambda)-\sigma_{R C P}(\lambda)\right)}{\sigma_{L C P}(\lambda)+\sigma_{R C P}(\lambda)}
$$

Nanoparticles synthesis and dimerization. Gold bipyramids (BPs) were synthesized following the procedure by Sánchez-Iglesias et al. ${ }^{8}$, although we replaced citric acid with sodium citrate during seed preparation. SEED. Briefly, $14.71 \mathrm{mg}$ of trisodium citrate was dispersed in $9.338 \mathrm{~mL}$ of ultrapure water (conductivity $<0.06 \mu \mathrm{S}$ ) and then $3.91 \mu \mathrm{L}$ of $0.6347 \mathrm{M} \mathrm{HAuCl}_{4}$ was added. Next, $661 \mu \mathrm{L}$ of 25 wt \% CTAC in water was added to the mixture under gentle stirring and $250 \mu \mathrm{L}$ of $0.025 \mathrm{M} \mathrm{NaBH}_{4}$. The color change (from yellow to brown) was observed. After 2 minutes of vigorous stirring, the vial was closed and solution was heated in oil bath at $80^{\circ} \mathrm{C}$ for 90 minutes under stirring, leading to color change from brown to red. The seed solution was stored in room temperature. BPs GROWTH. $50 \mathrm{~mL}$ of $0.1 \mathrm{M}$ CTAB was mixed with $2.5 \mathrm{~mL}$ of $0.01 \mathrm{M} \mathrm{HAuCl}_{4}$ solution. Next, $0.5 \mathrm{~mL}$ of $0.01 \mathrm{M} \mathrm{AgNO}_{3}, 1 \mathrm{~mL}$ of $1 \mathrm{M} \mathrm{HCl}$ and $0.4 \mathrm{~mL}$ of $0.1 \mathrm{M}$ ascorbic acid were added. Certain volume of gold seeds $(0.1-2.5 \mathrm{~mL})$ was added to the aqueous solution. The mixture was gently mixed and left in $30^{\circ} \mathrm{C}$ for at least 2 hours. Then extinction spectra were measured. DIMERISATION. It was conducted following the experimental procedure by Malachosky and Guyot-Sionnest ${ }^{9}$. Briefly, $125 \mu \mathrm{L}$ of gold nanoparticles solution was placed in a $4 \mathrm{~mL}$ cuvette and mixed with $2.725 \mathrm{~mL}$ of ultrapure water, $100 \mu \mathrm{L}$ of $30 \mathrm{mM}$ $\mathrm{HCl}$ solution (final volume $3 \mathrm{~mL}$ and $\mathrm{pH} 3$ ). UV/Vis spectrum was measured instantly. Then, $50 \mu \mathrm{L}$ of $6 \mathrm{mM}$ L-cysteine solution was added and extinction spectra were collected in 10-minute intervals to observe the progress of BPs binding.

Transmission electron microscopy. $1.5 \mathrm{~mL}$ of the solution after dimerization was transferred into $2 \mathrm{~mL}$ Eppendorf tube and centrifuged (4000 RCF, 10 minutes, 3 times) to remove supernatant containing most of the non-bound L-cysteine and excess CTAB. Pellet was re-dispersed in $5 \mathrm{~mL}$ of ultrapure water for storage. Solution containing washed gold bipyramids, dimers and oligomers was diluted 100 times to achieve separation of nanoparticles before manual drop-casting $(10 \mu \mathrm{L})$ onto TEM grids (Agar Scientific, S160). TEM imaging was performed on Hitachi H-800 system under 150 kV where various mono-, di- and oligomers were found separated. 


\section{Figures}

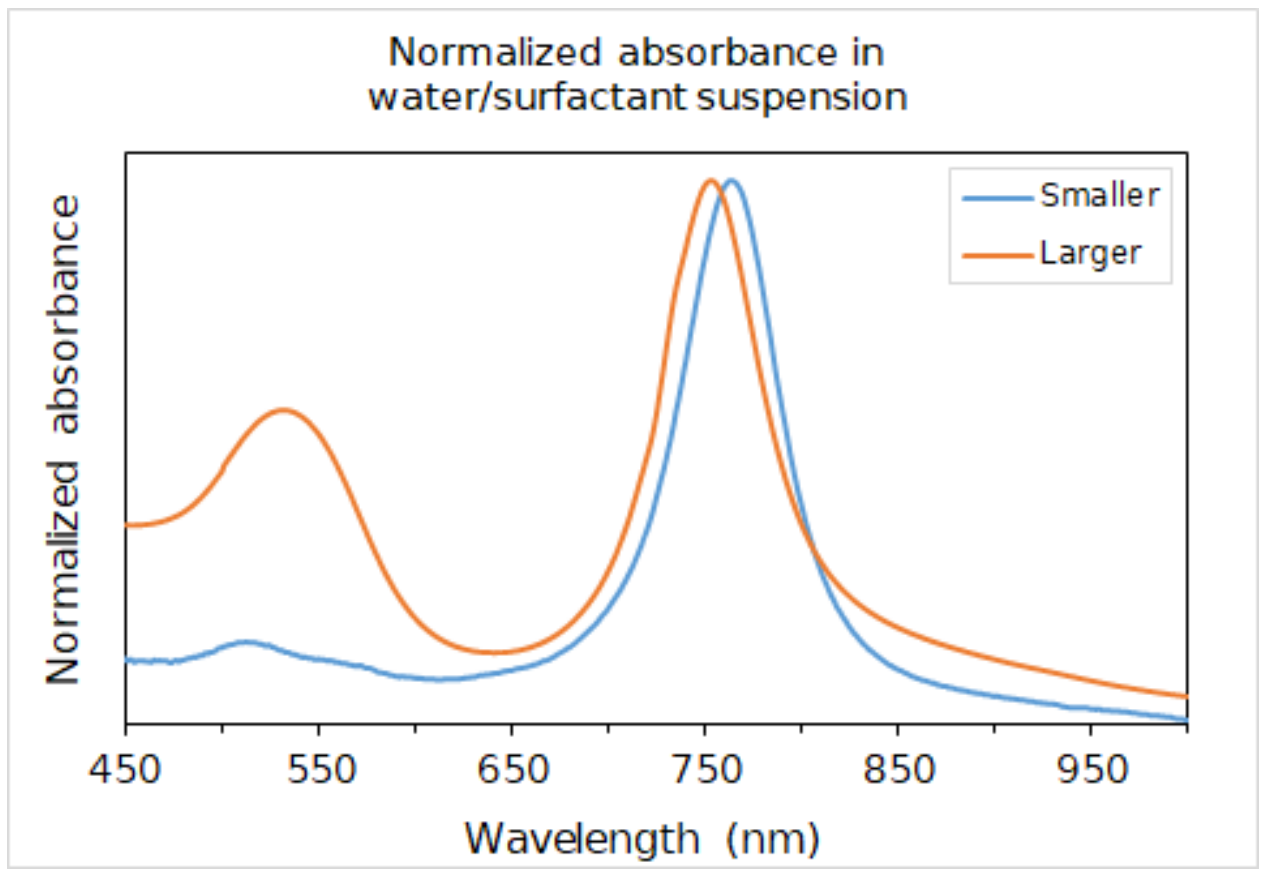

Figure S1 Absorbance spectra of nanobipyramids suspended in water/surfactant mixture. Labels "Larger" and "Smaller" in the legends refer to nanobipyramids in Figure S2 and Figure S3, respectively. 

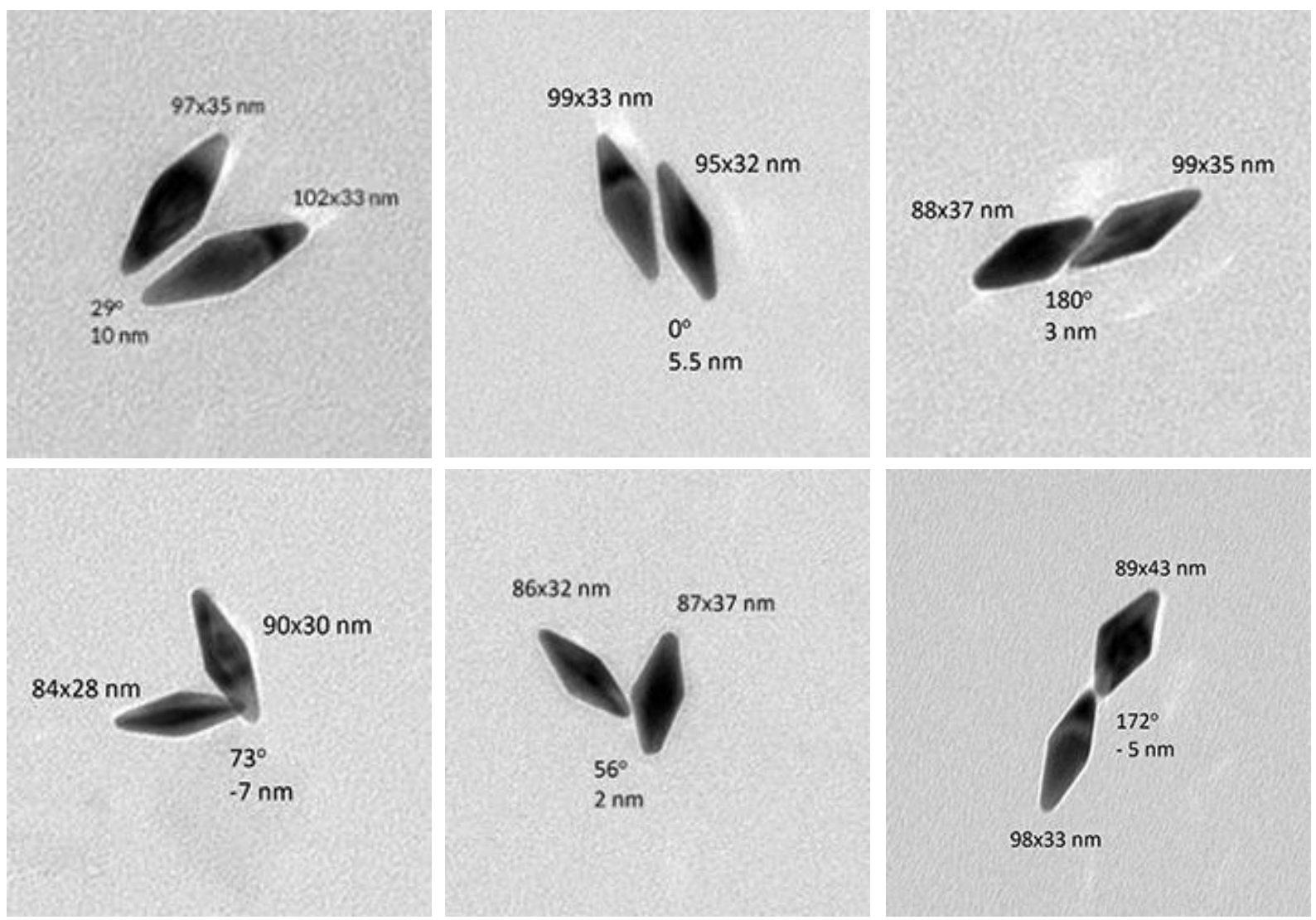

Figure S2 TEM images of nanobipyramids dimers - Sample with LSPR $751 \mathrm{~nm}$. Average dimensions $93 \pm 7 \times 35 \pm 4 \mathrm{~nm}$. Indicated dimensions include long axis, short axis, non-reflex angle between long axes and distance (as defined in the main text).
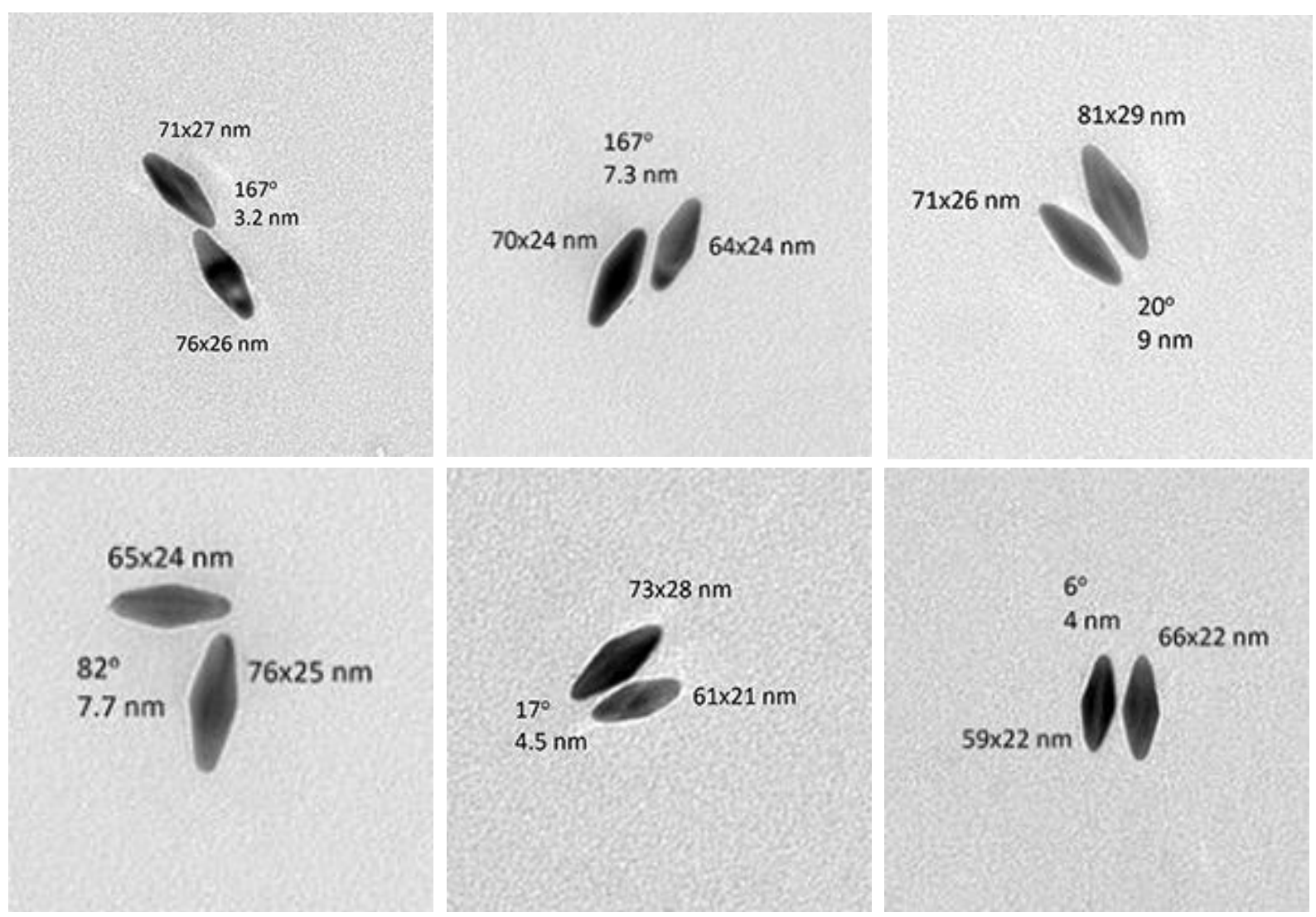

Figure S3 TEM images of nanobipyramids dimers - Sample with LSPR $764 \mathrm{~nm}$. Average dimensions $71 \pm 6 \times 27 \pm 3 \mathrm{~nm}$. Indicated dimensions include long axis, short axis, non-reflex angle between long axes and distance (as defined in the main text). 

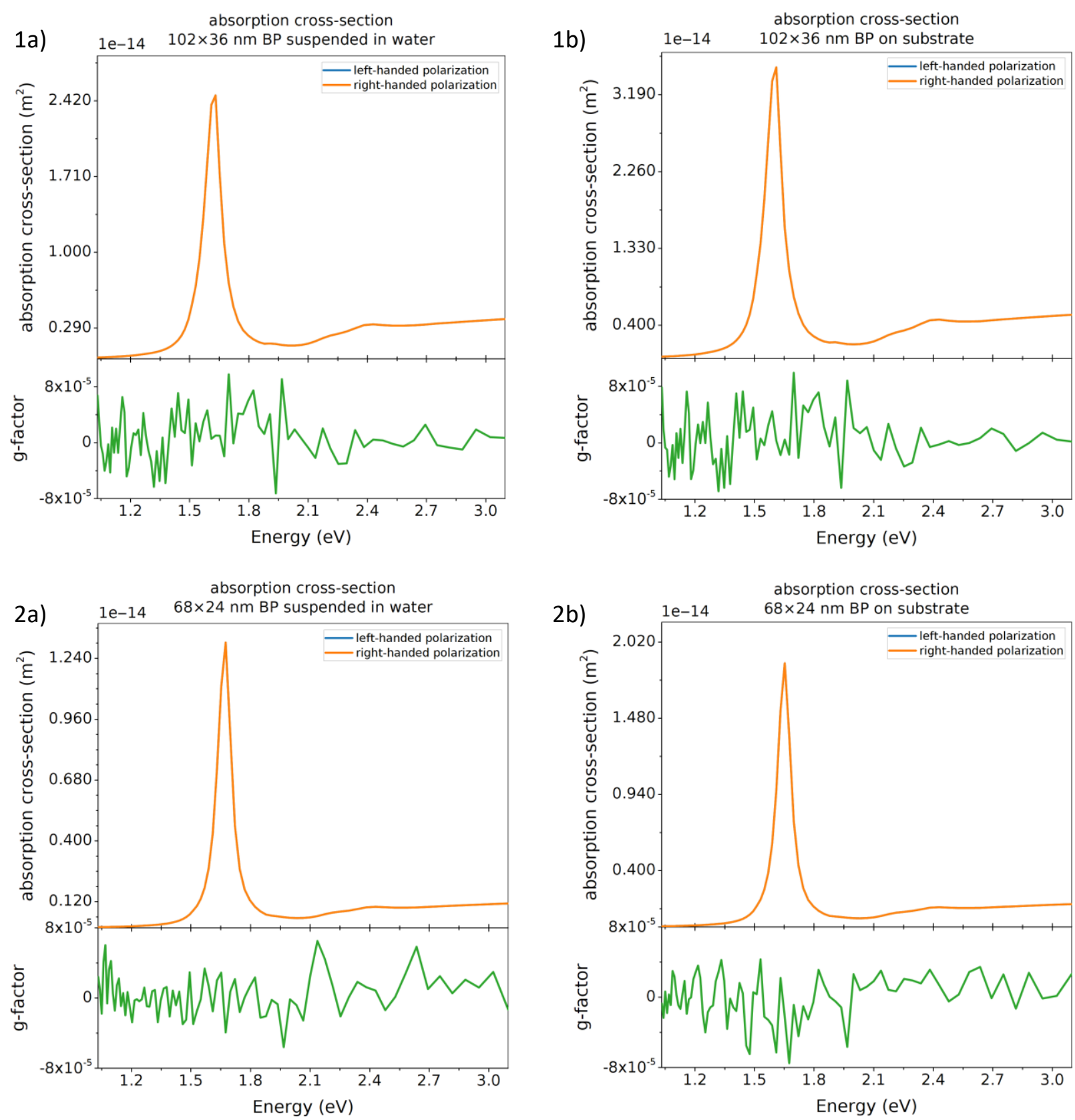

Figure S4 Absorption spectra of $102 \times 36 \mathrm{~nm}(1 \mathrm{a}-\mathrm{b})$ and $68 \times 24 \mathrm{~nm}(2 \mathrm{a}-\mathrm{b})$ single nanobipyramid suspended in water (1-2a) and on substrate (1-2b) with long axis at $31^{\circ}$ pitch relative to excitation wavefront. Lorentzian fit in LSPR range of larger particle-on-substrate spectrum yields maximum at $1.597 \mathrm{eV}(775 \mathrm{~nm})$ with FWHM of $\sim 0.103 \mathrm{eV}(50 \mathrm{~nm})$. Blue line for lefthanded polarization in each spectrum is overlapped with orange line for right-handed spectrum. G-factor (defined in the main text) spectra indicate dissymmetry coming merely from numerical noise. 
1a)

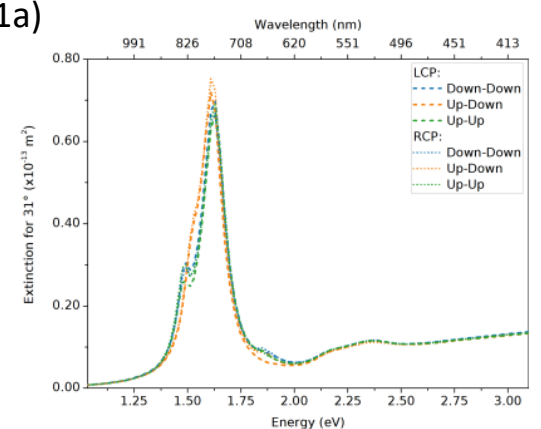

2a)

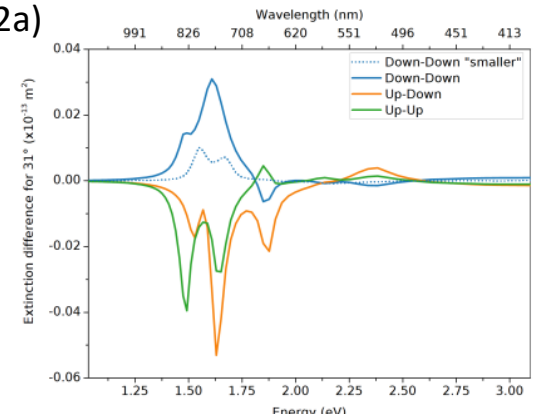

3a)

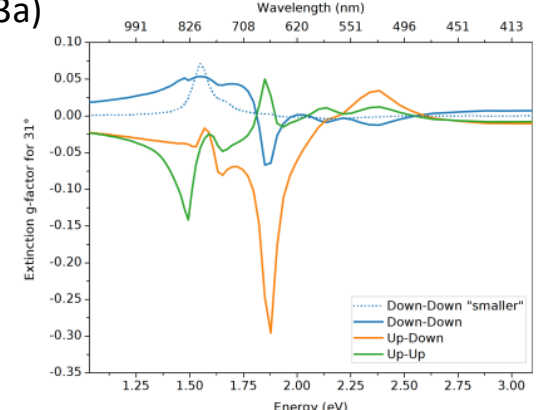

1b)

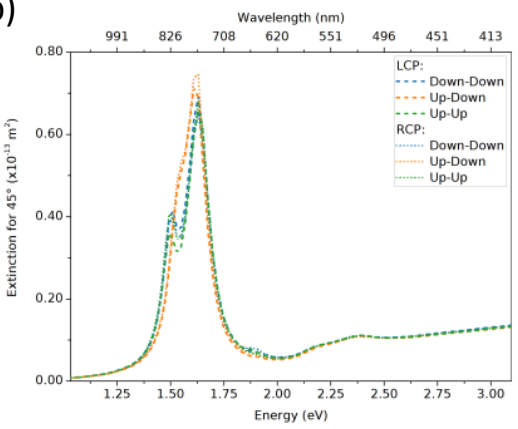

2b)

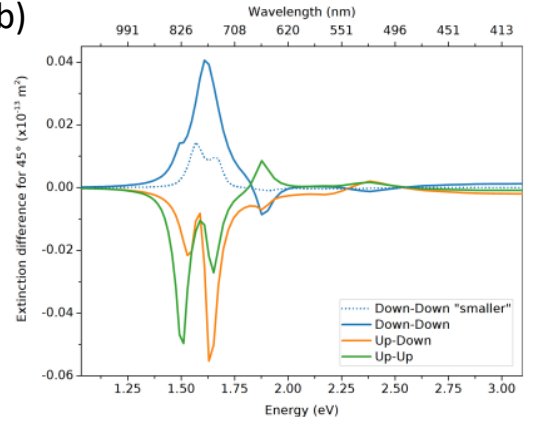

3b)

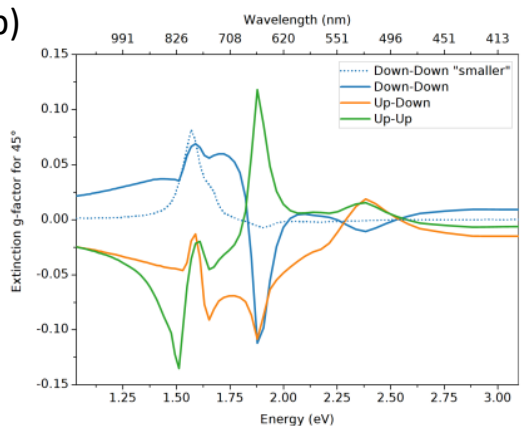

1c)

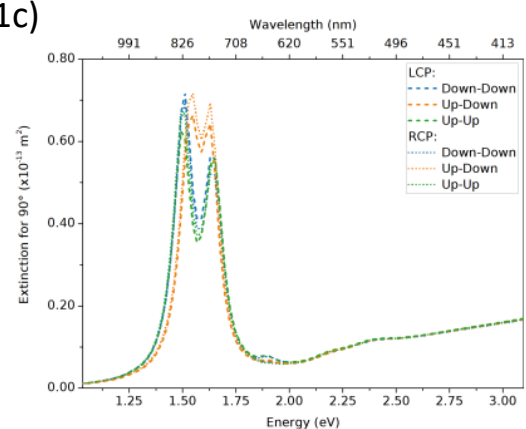

2c)

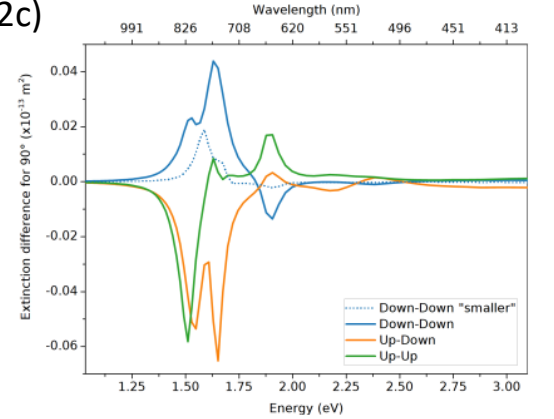

3c)

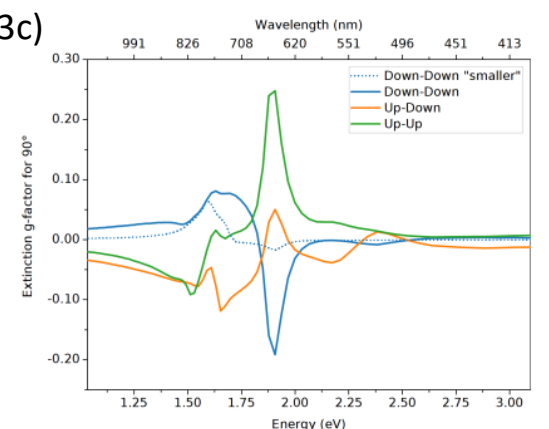

1d)

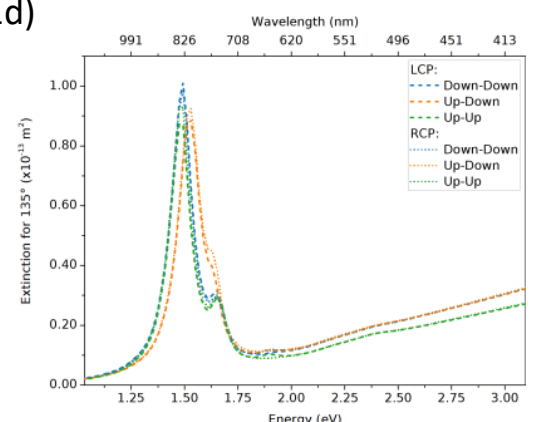

2d)

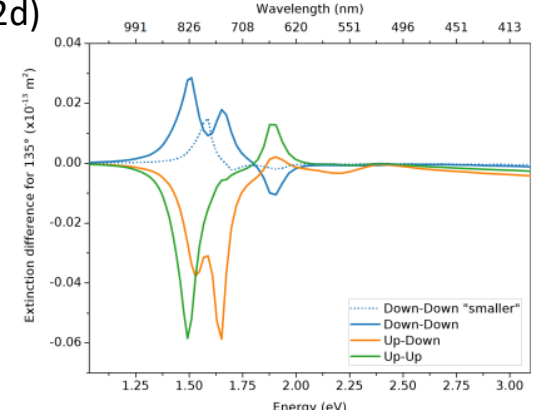

3d)

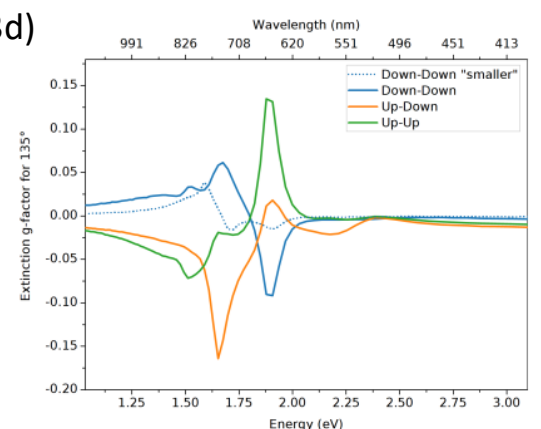

1e)

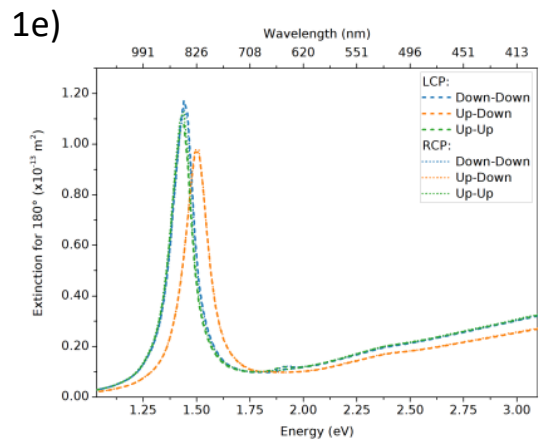

2e)

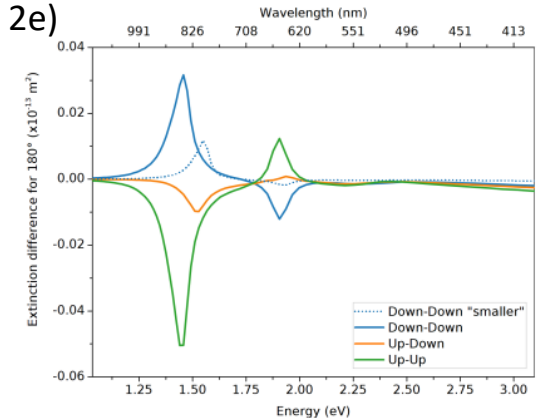

$3 e)$

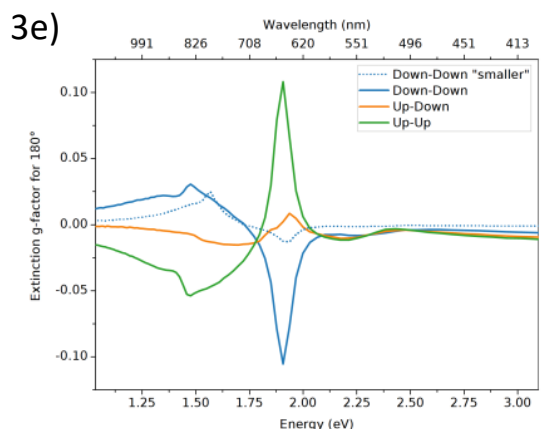

Figure S5 Extinction in 102x36 nm BP dimers as excited by circularly polarized light (1a-e), absolute extinction difference in BP dimers as excited by circularly polarized light (2ae), and relative extinction difference (g-factor) of BP dimers as excited by circularly polarized light (3a-e). Absolute and relative extinction difference spectra for Down-Down configuration of $68 \times 24 \mathrm{~nm}$ BP dimers are presented as dotted lines in rows 2 and 3 . 
1a)

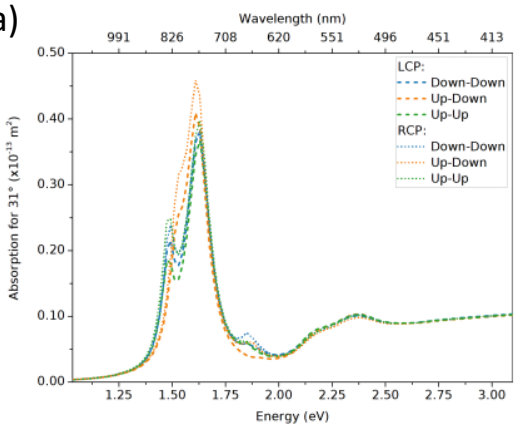

2a)

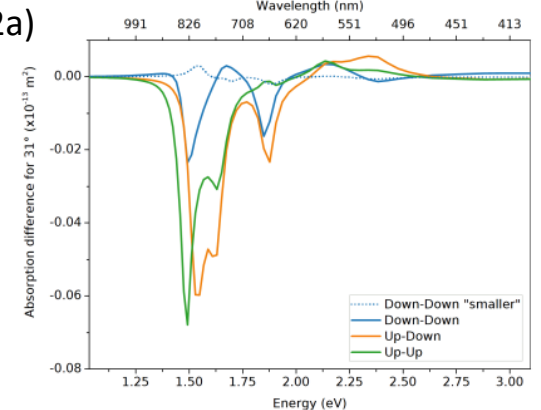

3a)

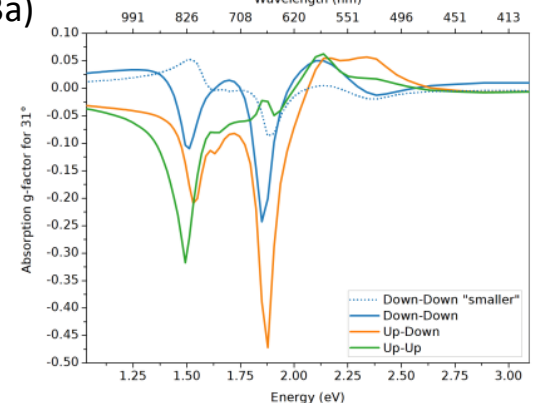

1b)

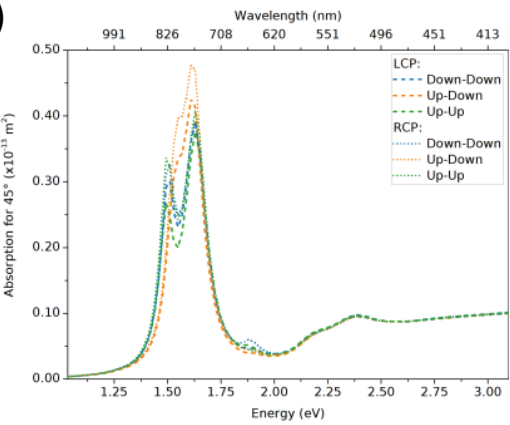

2b)

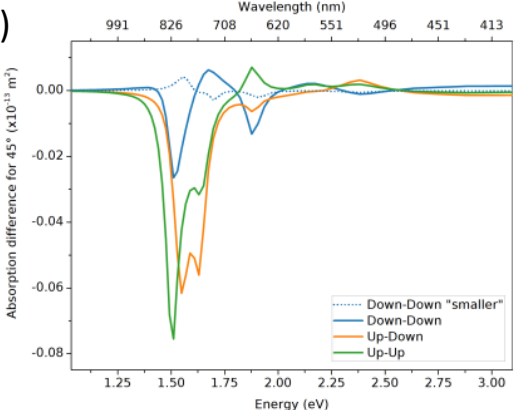

3b)

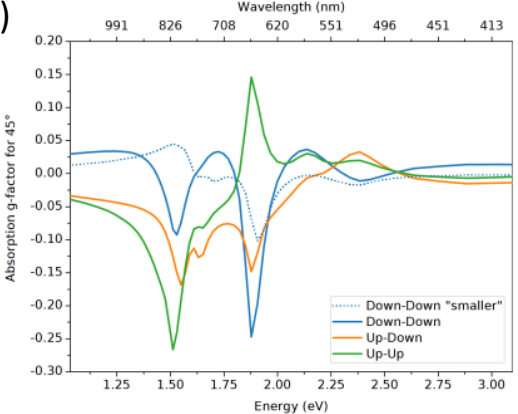

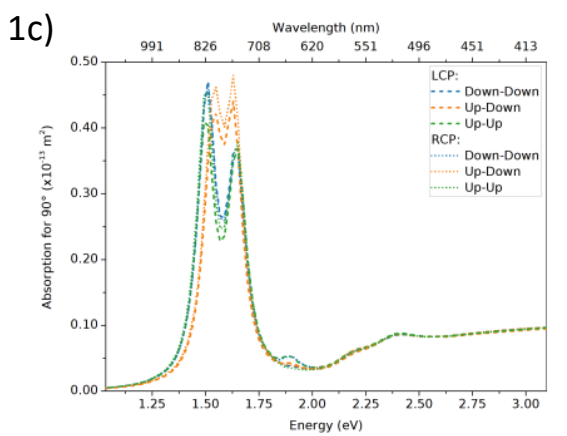

1d)
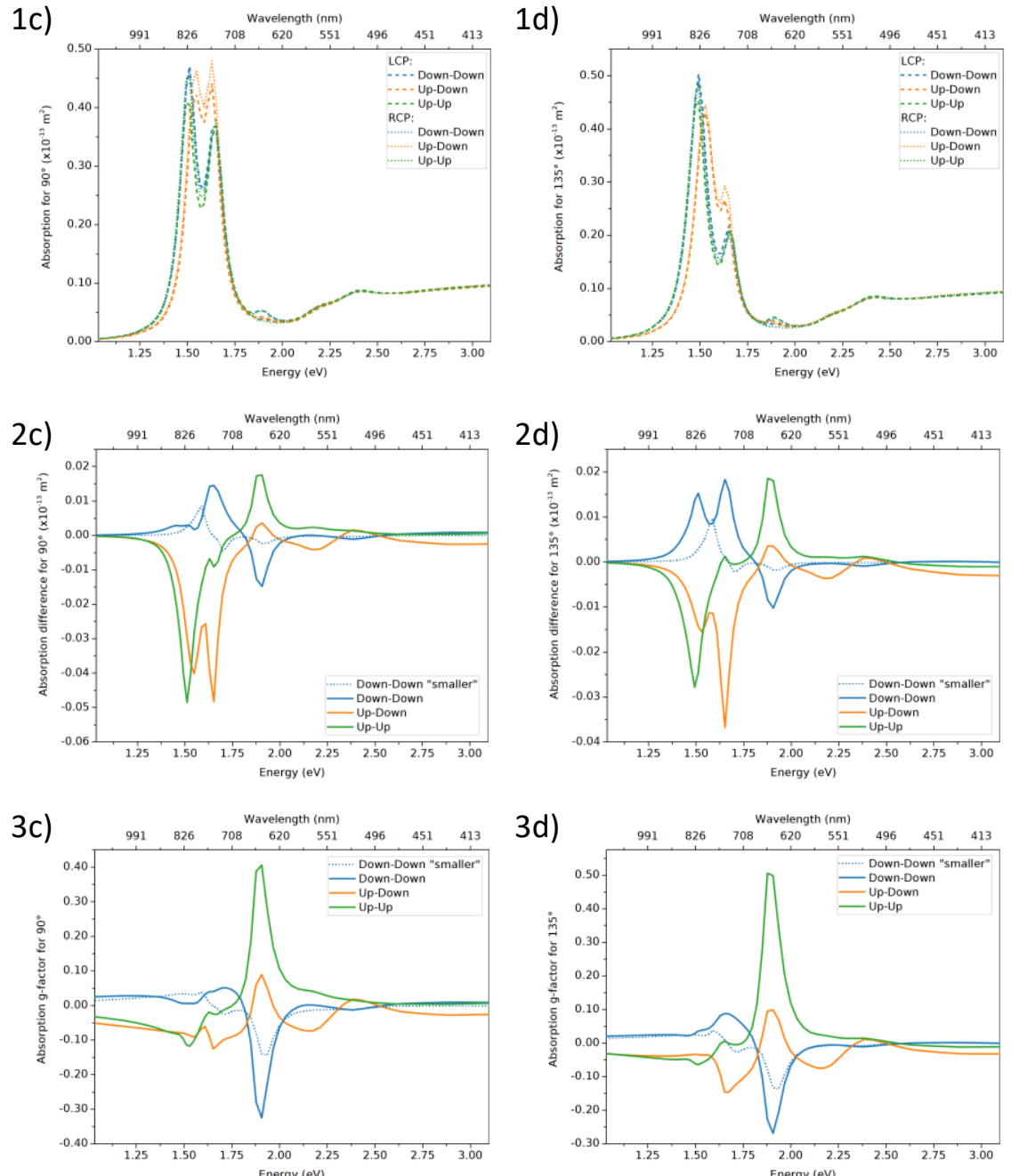

3d)

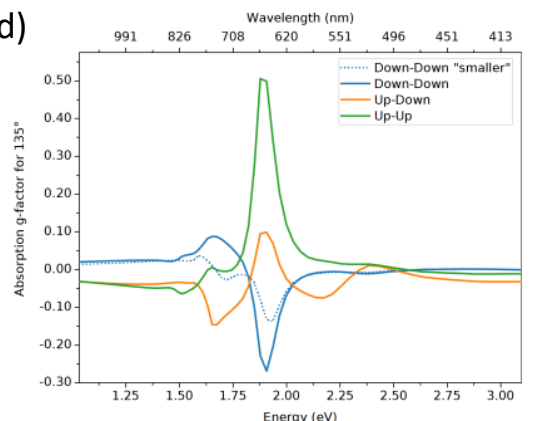

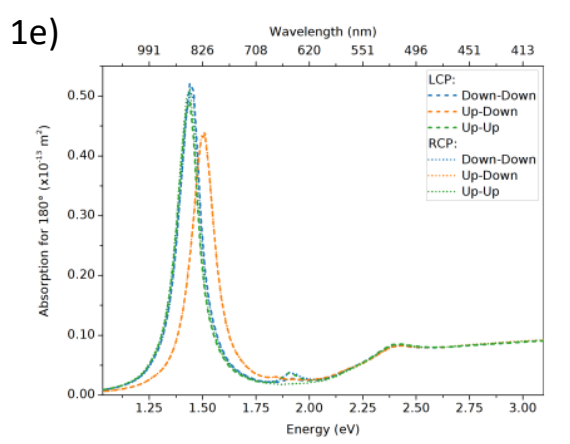
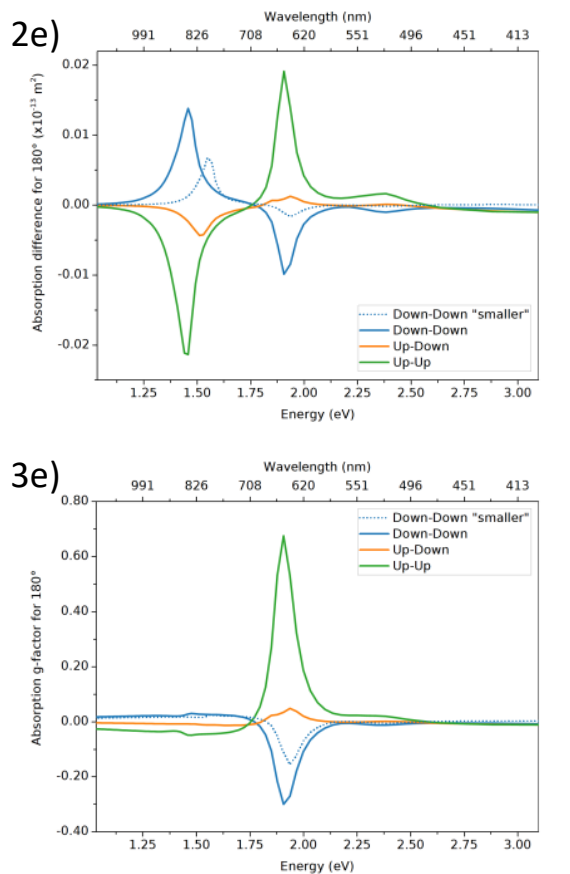

Figure S6 Absorption in 102x36 nm BP dimers as excited by circularly polarized light (1a-e), absolute absorption difference in BP dimers as excited by circularly polarized light (2ae), and relative absorption difference (g-factor) of BP dimers as excited by circularly polarized light (3a-e). Absolute and relative absorption difference spectra for Down-Down configuration of $68 \times 24 \mathrm{~nm}$ BP dimers are presented as dotted lines in rows 2 and 3 . 
1a)

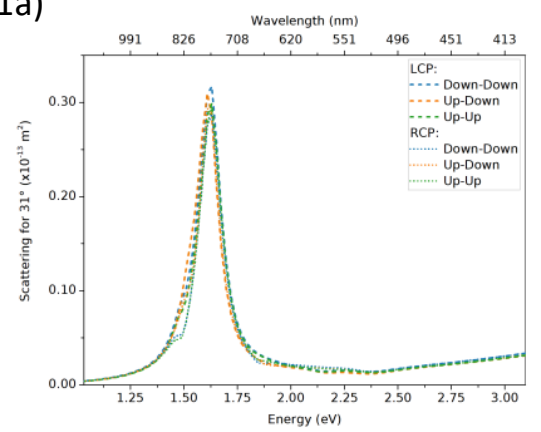

2a)

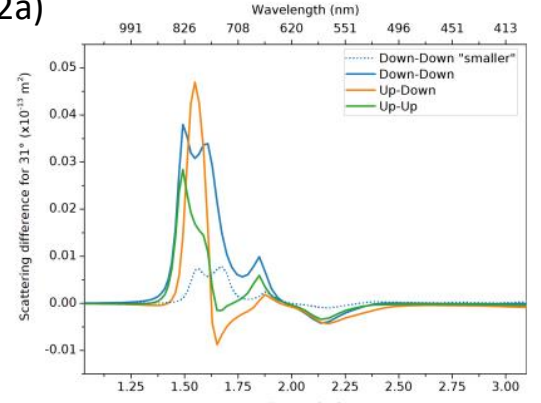

3a)

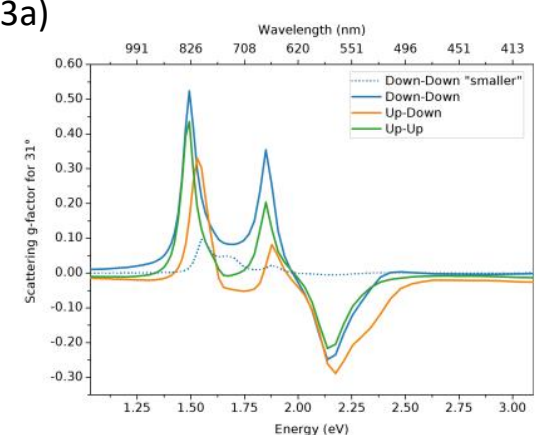

1b)

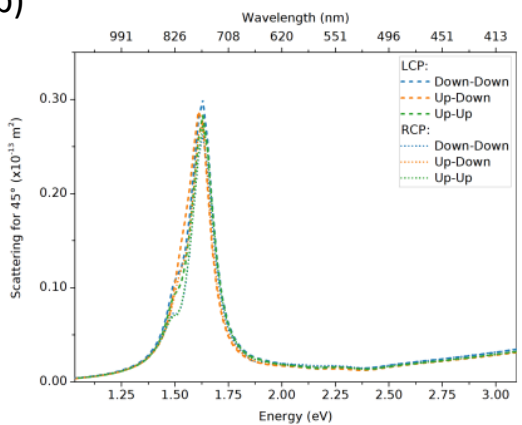

2b)

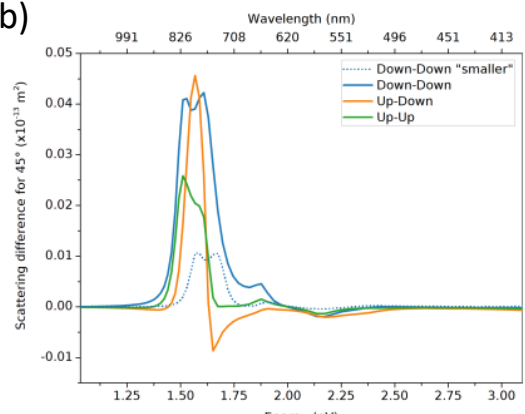

3b)

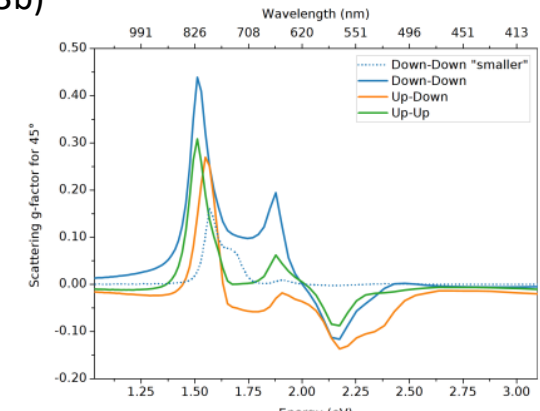

1c)

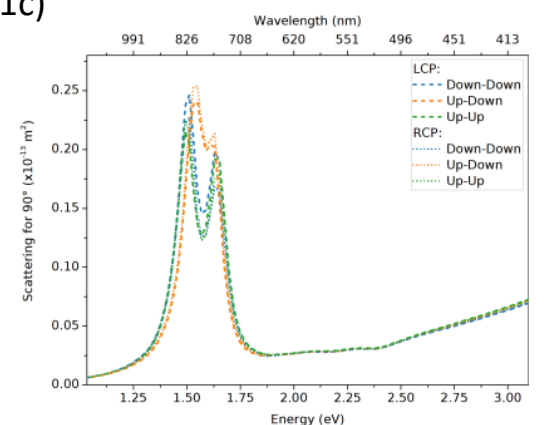

2c)

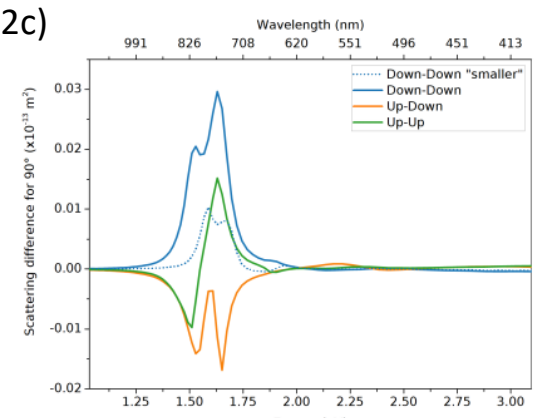

3c)

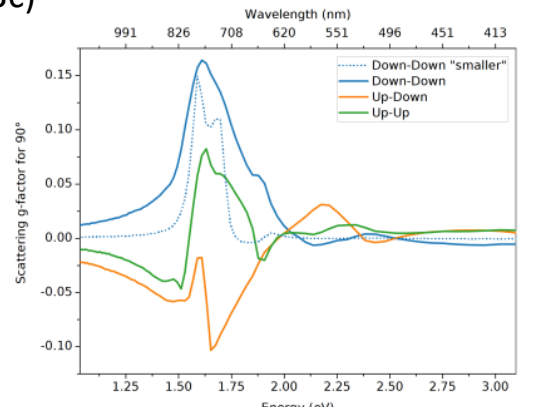

1d)

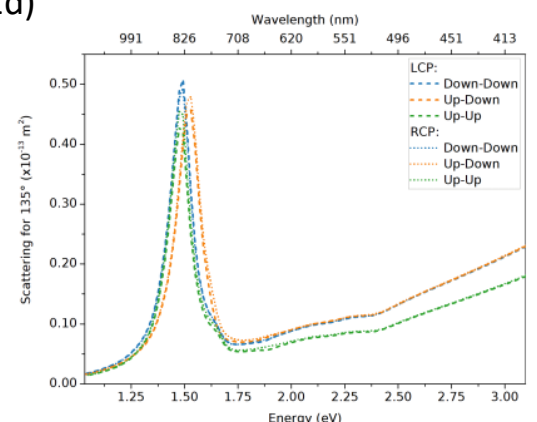

2d)

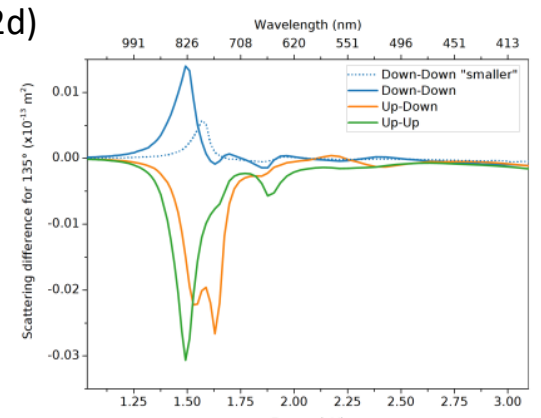

3d)

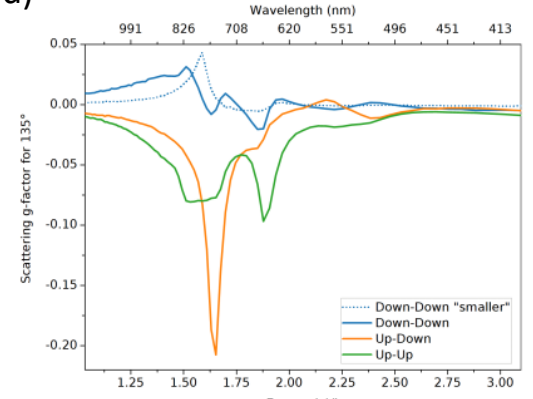

1e)

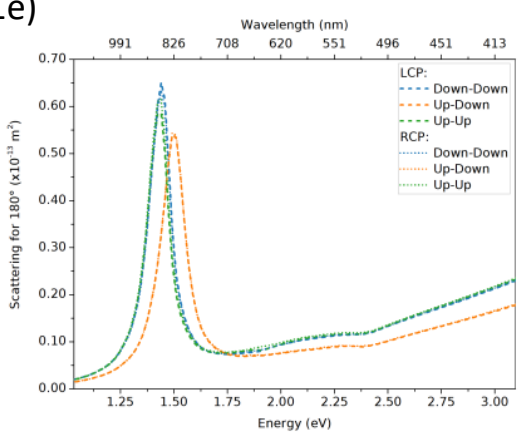

2e)

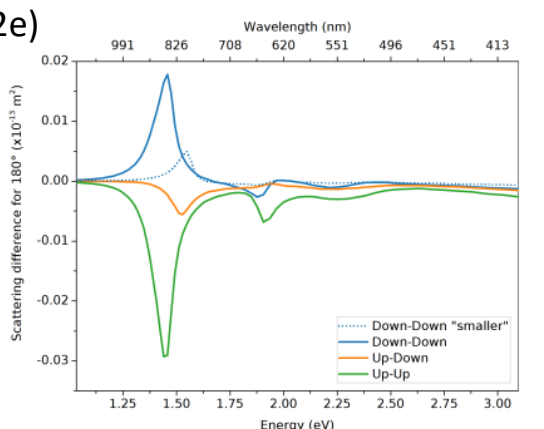

$3 e)$

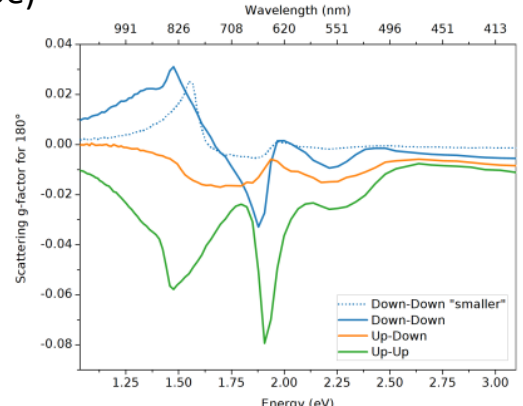

Figure S7 Scattering in 102x36 nm BP dimers as excited by circularly polarized light (1a-e), absolute scattering difference in BP dimers as excited by circularly polarized light (2ae), and relative scattering difference (g-factor) of BP dimers as excited by circularly polarized light (3a-e). Absolute and relative scattering difference spectra for Down-Down configuration of $68 \times 24 \mathrm{~nm}$ BP dimers are presented as dotted lines in rows 2 and 3 . 
a)

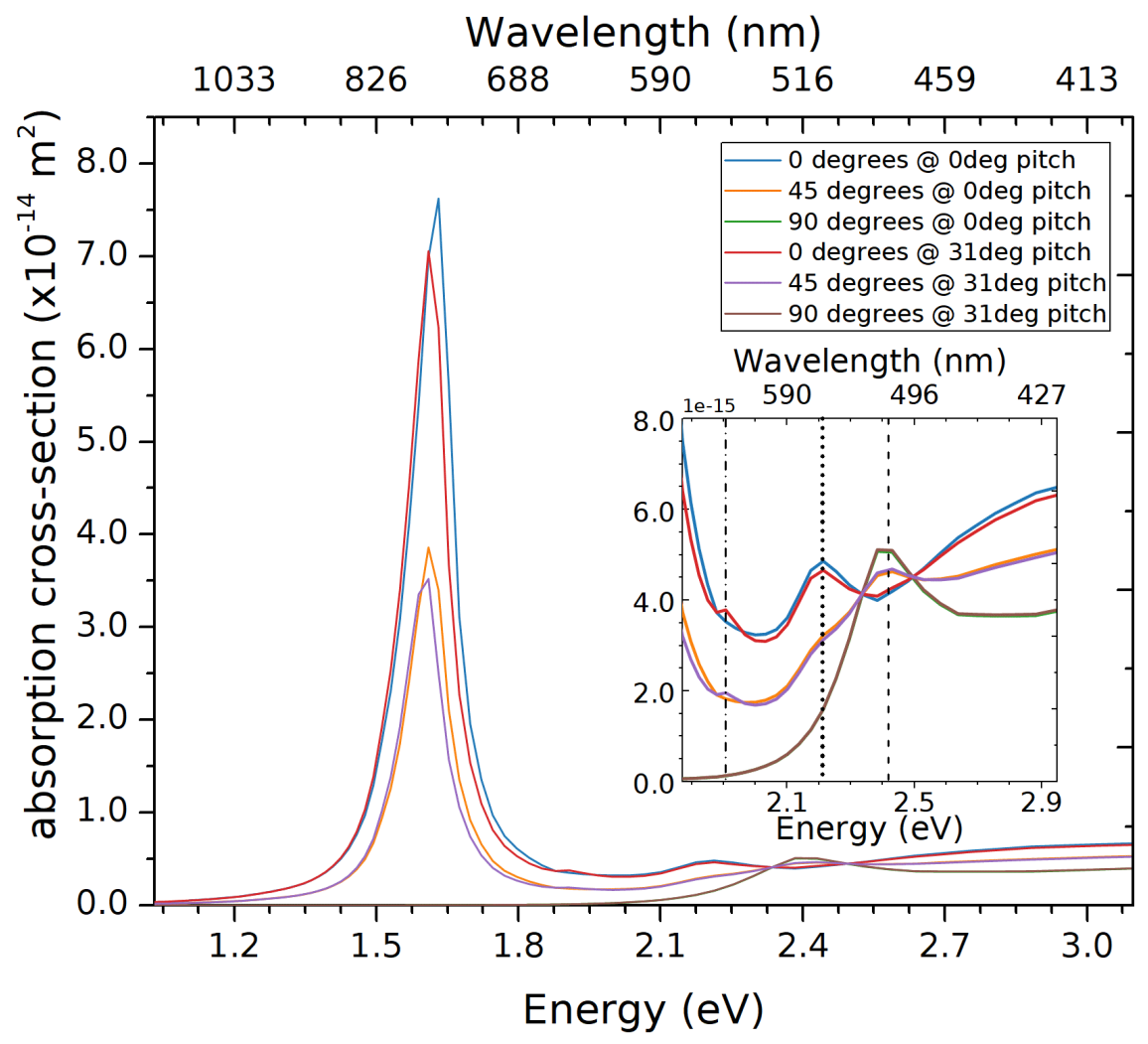

b)

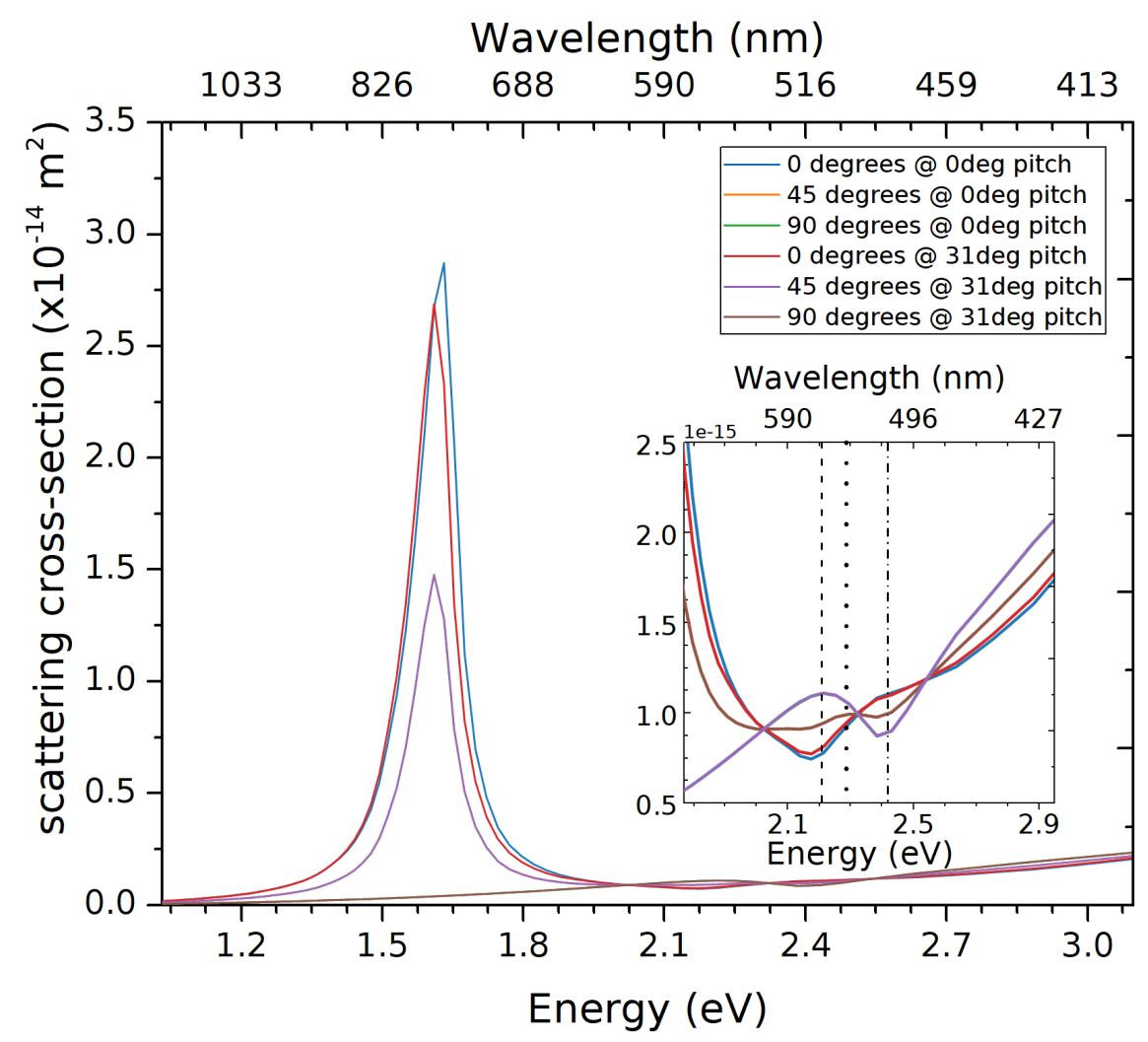

Figure S8 Absorption (a) and scattering (b) cross-sections of single gold bipyramid excited with electromagnetic wave linearly polarized parallel to projection of long $\left(0^{\circ}\right)$ and short $\left(90^{\circ}\right)$ axes of single $102 \times 36 \mathrm{~nm} \mathrm{BP}$ onto wavefront plane. Wave vector is perpendicular to long axis $\left(0^{\circ}\right.$ pitch $)$ and at an angle to long axis $\left(31^{\circ}\right.$ pitch resulting from BP lying on one side on substrate). Insets show close-ups on low-amplitude cross-sections in high-energy region. 

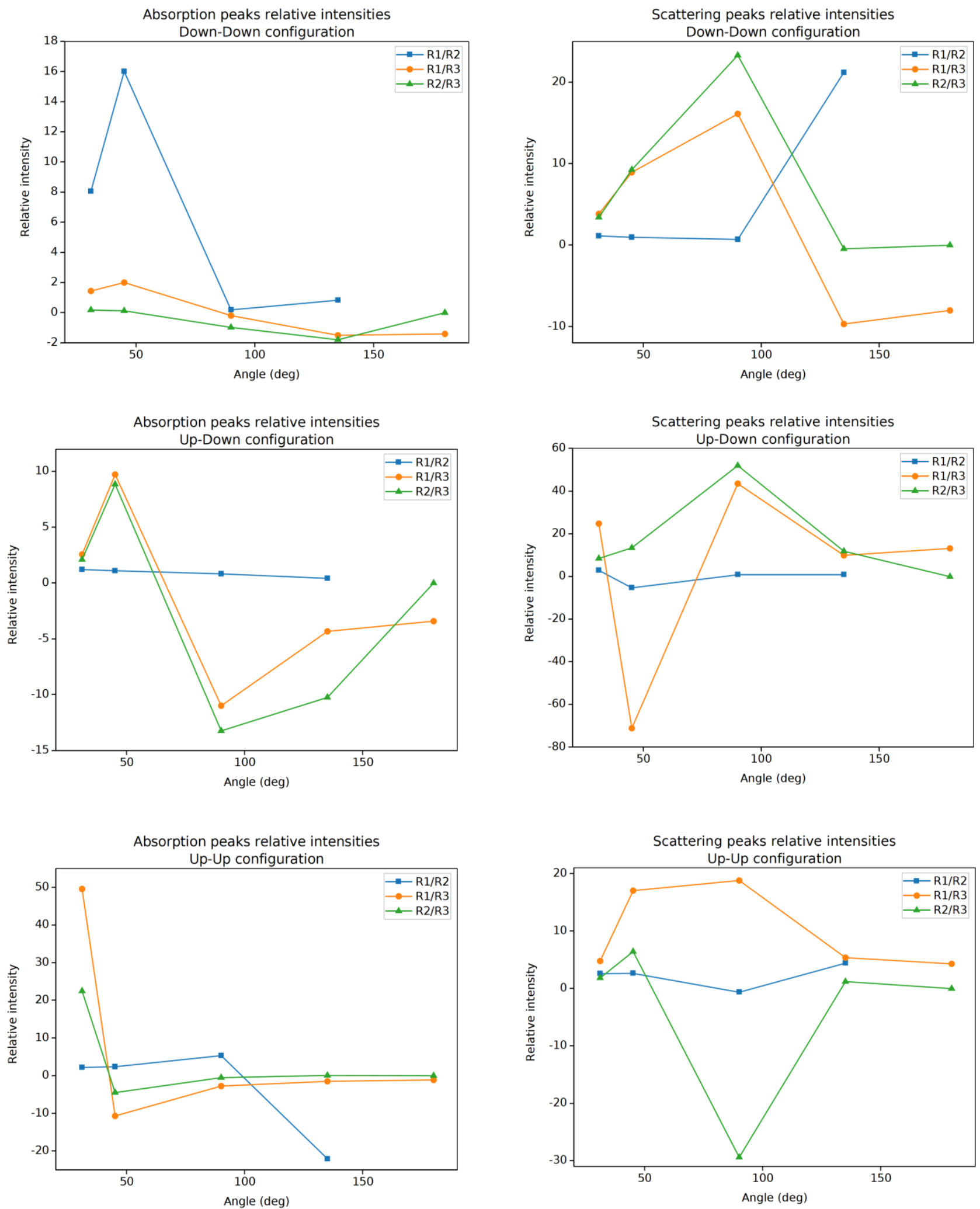

Figure S9 Relative intensities of three lowest-energetic resonances differences for absorption and scattering differential spectra of 102x36 nm BP dimers. R1, R2, and R3 refer to respective resonance amplitude difference at resonances: $\sim 1.5 \mathrm{eV}, \sim 1.65 \mathrm{eV}$, and $\sim 1.9 \mathrm{eV}$, respectively. The distance between bipyramids is $5 \mathrm{~nm}$ as defined in the main text. 


\section{References}

(1) Bashkatov, A. N.; Genina, E. A., Water Refractive Index in Dependence on Temperature and Wavelength: A Simple Approximation; SPIE, 2003; Vol. 5068.

(2) Lide, D. R., CRC Handbook of Chemistry and Physics, 85th Edition; Taylor \& Francis, 2004.

(3) Schiebener, P.; Straub, J.; Sengers, J. M. H. L.; Gallagher, J. S., Erratum: Refractive Index of Water and Steam as Function of Wavelength, Temperature and Density [J. Phys. Chem. Ref. Data 19, 677 (1990)]. Journal of Physical and Chemical Reference Data 1990, 19, 1617-1617, DOI: $10.1063 / 1.555850$.

(4) Johnson, P. B.; Christy, R. W., Optical Constants of the Noble Metals. Physical Review B 1972, 6, 4370-4379, DOI: 10.1103/PhysRevB.6.4370.

(5) Sassaroli, E.; Li, K. C. P.; O'Neill, B. E., Numerical Investigation of Heating of a Gold Nanoparticle and the Surrounding Microenvironment by Nanosecond Laser Pulses for Nanomedicine Applications. Physics in Medicine and Biology 2009, 54, 5541-5560, DOI: 10.1088/0031-9155/54/18/013.

(6) Yushanov, S.; Crompton, J. S.; Koppenhoefer, K. C., Mie Scattering of Electromagnetic Waves. In Proceedings of the 2013 COMSOL Conference in Boston, COMSOL Conference, 2013. URL: https://www.comsol.com/paper/download/181101/crompton paper.pdf

(7) Sivun, D.; Vidal, C.; Munkhbat, B.; Arnold, N.; Klar, T. A.; Hrelescu, C., Anticorrelation of Photoluminescence from Gold Nanoparticle Dimers with Hot-Spot Intensity. Nano Letters 2016, 16, 7203-7209, DOI: 10.1021/acs.nanolett.6b03562.

(8) Sánchez-Iglesias, A.; Winckelmans, N.; Altantzis, T.; Bals, S.; Grzelczak, M.; Liz-Marzán, L. M., High-Yield Seeded Growth of Monodisperse Pentatwinned Gold Nanoparticles through Thermally Induced Seed Twinning. Journal of the American Chemical Society 2016, 139, 107110, DOI: $10.1021 /$ jacs.6b12143.

(9) Malachosky, E. W.; Guyot-Sionnest, P., Gold Bipyramid Nanoparticle Dimers. J Phys Chem $C$ 2014, 118, 6405-6412, DOI: 10.1021/jp412409u. 